\title{
Análisis sobre la percepción frente al riesgo por inundación en dos barrios de Concepción del Uruguay, Entre Ríos, Argentina
}

\author{
GRISELDA ELENA CARÑEL ${ }^{1,2,3 ;}$ ROSANA SILVINA BONNIN"; FRANCISCO SAVOY1,4 \\ ${ }^{1}$ Facultad de Ciencias de la Salud, Universidad Nacional de Entre Ríos, \\ Argentina. \\ ${ }^{2}$ Facultad de Humanidades, Urbanismo y Ciencias, Universidad Nacional del \\ Litoral, Argentina. \\ ${ }^{3}$ Facultad de Humanidades, Artes y Ciencias Sociales, Universidad Autónoma \\ de Entre Ríos, Argentina. \\ ${ }^{4}$ CONICET. \\ gecargnel@yahoo.com.ar; rosa_bonnin@hotmail.com; fransavoy@hotmail.com
}

\section{RESUMEN}

En los últimos 35 años, en la región del Litoral argentino, casi un millón de personas han sido evacuadas en inundaciones; estadísticamente uno de cada I3 habitantes ha sido afectado. Muchos actores sociales, a pesar de haber vivido situaciones de inundación, tienden a negar las consecuencias o a menospreciar la ocurrencia del fenómeno. Este trabajo buscó identificar y caracterizar la amenaza por inundación, conocer la población en riesgo y la percepción y el comportamiento o conducta que poseen los habitantes de los barrios Cantera 25 y San José y los funcionarios locales de la ciudad de Concepción del Uruguay frente al riesgo por inundación. Se emplearon técnicas cuantitativas y cualitativas y los análisis espaciales se realizaron mediante un SIG. Se realizaron iso encuestas en marzo y abril de 2013 y entrevistas semiestructuradas a funcionarios, concejales de la Municipalidad; representantes de Defensa Civil y de la Cruz Roja.

PALABRAS CLAVE: percepción del riesgo; inundación; vulnerabilidad; desastres. 


\section{ABSTRACT}

\section{ANALYSIS OF PERCEPTION AGAINST FLOOD RISK IN TWO NEIGHBORHOODS OF}

CONCEPCIÓN DEL URUGUAY, ENTRE RIOS, ARGENTINA

There have been nearly a million people evacuated within the past 35 years in the Litoral Región of Argentina as a consequence of floods - that means that one out of every 13 citizens have been affected. Many social actors and institutions, despite having been victims of floods, tend to deny and undervalue the consequences of these events. The aim of this work was to identify and assess the flood threat, survey the population under risk and survey the perception and conduct in the event of floods of the local government officials and of the residents in the Cantera 225 and San José neighbourhoods in Concepción del Uruguay. Quantitative and qualitative techniques were used. Spatial analysis was carried out in GIS. One-hundred and fifty surveys and semi-structured interviews were made In March and April 2013 to government officials, city councilmen, Civil Defence and International Red Cross representatives.

KEYWORDS: risk perception; flood; vulnerability; disasters. 


\section{RESUMO}

\section{ANÁLISE SOBRE A PERCEPÇÃO DE RISCO CONTRA INUNDAÇÕES EM DOIS BAIRROS DE CONCEPCIÓN DEL URUGUAY, ENTRE RÍOS, ARGENTINA}

Nos últimos 35 anos, na região do litoral da Argentina, quase um milhão de pessoas foram evacuadas em inundações; estatisticamente uma em cada 13 pessoas tem sido afetada. Muitos atores sociais, apesar de ter vivido em situaçôes de inundação, tendem a negar ou menosprezar as conseqüências da ocorrência do fenômeno. Este estudo buscou identificar e caracterizar o perigo de inundação, conhecer a população em risco e conhecer a percepção e conduta das autoridades locais e dos habitantes de Cantera 225 e San Jose distritos na cidade de Concepción del Uruguay contra o risco de dilúvio. Foram utilizadas técnicas quantitativas e qualitativas e os análises espaciais foram realizadas utilizando um SIG. I5o inquéritos e entrevistas semi-estruturadas foram feitas em março e abril de 2013 com funcionários, vereadores do Município, representantes da Defesa Civil e Cruz Vermelha.

PALAVRAS-CHAVE: percepção de risco; inundação; vulnerabilidade; desastres. 


\section{Introducción}

Cotidianamente, se habla en la provincia de Entre Ríos de los «desastres» de las inundaciones que periódicamente se producen por la crecida de alguno de los ríos y arroyos que surcan la misma; así como del «riesgo» a que está sometida la población y a la asistencia de socorro prestada por el Estado. Ahora bien, ¿en qué medida se conoce de qué se habla? ¿Se piensa en lo mismo al decir iguales palabras?

En el Marco de Acción de Hyogo (MAH), ${ }^{1}$ que es el instrumento más importante para la implementación de la reducción del riesgo de desastres que adoptaron los Estados miembros de las Naciones Unidas, se fija como objetivo general «aumentar la resiliencia de las naciones y las comunidades ante los desastres al lograr, para el año 2015, una reducción considerable de las pérdidas que ocasionan los desastres, tanto en términos de vidas humanas como en cuanto a los bienes sociales, económicos y ambientales de las comunidades y los países». El MAH ofrece tres objetivos estratégicos y cinco áreas prioritarias para la toma de acciones, al igual que principios rectores y medios prácticos para aumentar la resiliencia de las comunidades vulnerables a los desastres, en el contexto del desarrollo sostenible.

Con estas premisas y con la creencia de que el «riesgo de desastre» implica la probabilidad de daños socioeconómicos ante la ocurrencia de un evento físico ya sea de carácter natural o antrópico, se encaró este trabajo sobre la percepción del riesgo que poseen los habitantes de Concepción del Uruguay.

Para eso se adoptó una perspectiva teórica desde la cual las inundaciones se caracterizan por ser eventos riesgosos de tipo «artificial», o más bien, «no natural», es decir que asocian su presencia más a un avance vertiginoso del sistema urbano que a un funcionamiento catastrófico propio del sistema natural (Burton y Kates, 1972).

Se puede conceptualizar el riesgo natural como aquellos acontecimientos ambientales que exceden la capacidad normal de ajuste y amortización de los sistemas humanos para absorberlos, o bien como una alteración sustantiva de la vinculación permanente que mantiene el hombre con su entorno biofísico y que, casi necesariamente, se asocia a la idea

1 EIRD (2005). Marco de acción de Hyogo para 2005-2015. Aumento de la resiliencia de las naciones y las comunidades ante los desastres. Disponible en: www.unisdr.org 
de daños. En este sentido, el problema de las inundaciones resulta claramente de un desajuste o disfunción, entre la sociedad y su entorno biofísico (Smith y Tobin, 1979; Ward, I978; Burton y Kates, 1972).

Según Rovira (1986), un aspecto importante de esta problemática resulta ser la percepción que el grupo humano tiene del evento y las acciones que se toman frente a ellas. Esto está influido tanto por la cultura como por la experiencia de cada individuo.

En ese aspecto, no hay que dejar de lado los estudios realizados luego de la catástrofe producida por el río Salado en la ciudad de Santa Fe en abril de 2003, destacándose los referidos a la construcción social del riesgo (Arrillaga et al., 2009; Masi y Kessler, 2009).

La ciudad de Concepción del Uruguay es la capital del Departamento Uruguay en la provincia de Entre Ríos. Está localizada en la margen occidental del Río Uruguay "el río de los pájaros», y a la vera de la Ruta Nacional I4, que la conecta a sólo $300 \mathrm{~km}$ con la Ciudad Autónoma de Buenos Aires a través del Complejo Ferrovial Zárate-Brazo Largo.

Tierra de patriotas como Urquiza y Ramírez, ciudad del Pronunciamiento libertario contra Rosas y protagonista de tantos otros hechos históricos de singular repercusión en el orden regional y nacional, fue fundada el 25 de junio de 1783 por don Tomás de Rocamora (Urquiza Almandóz, 2002). Fue capital de la provincia hasta fines del siglo XIX, por lo que cuenta con un rico patrimonio histórico cultural. Actualmente se ha convertido en una de las urbes más dinámicas de la provincia y con mayor crecimiento económico.

Sin embargo, la existencia de las actividades fabriles y de dársenas, históricamente ubicadas en la margen del río, han hecho que la ciudad se desarrolle de espaldas al borde costero. Más específicamente, la ciudad no se encuentra sobre el cauce del río Uruguay, sino sobre el Riacho Itapé, antigua desembocadura del arroyo Molino y del arroyo de la China, que luego se transformó en un brazo del río Uruguay al construirse el canal de acceso al puerto.

Si se le suman a su ubicación costera, precipitaciones de $1100 \mathrm{~mm}$ normales anuales y pavimentación en la mayor parte de su área urbana, las inundaciones y anegamientos son una amenaza constante.

La comprensión tanto del fenómeno inundación como de los daños que afectan y puedan afectar a la población, es fundamental para diseñar acciones preventivas o de contención. 
Calvo García-Torne (2009) señala que «esta comprensión es el elemento básico subyacente en los procesos de riesgo, ya que determinan, en buena medida, la selección y definición de prioridades».

Este trabajo, enmarcado en el PID-UNERioos $3,{ }^{2}$ tuvo como objetivo identificar la población en riesgo por inundación hídrica y la percepción, que de ella poseen tanto los habitantes de los barrios Cantera 25 y San José, como los funcionarios políticos de la ciudad de Concepción del Uruguay.

Conocer la percepción frente a un fenómeno, en este caso la inundación, resulta fundamental para elaborar y/o llevar adelante una mejor gestión del riesgo. Se intentó asimismo, apreciar en qué medida, la percepción del grupo humano se manifiesta en acción, opiniones y previsiones frente a los eventos riesgosos.

De acuerdo a lo señalado y como hipótesis con respecto a la población, podemos inferir que, si bien la gente afectada directamente reconoce la problemática como prioritaria, no tienen los medios culturales y económicos para trasladarse a lugares seguros.

\section{Materiales y métodos}

La ciudad de Concepción del Uruguay ( $32^{\circ} 29^{\prime} 04^{\prime \prime}$ S, 58 $14^{\circ} 13^{\prime \prime}$ O) se sitúa en el centro del corredor turístico del Río Uruguay. Entre el río y la ciudad se encuentra una serie de islas y zonas bajas anegables que forman parte del valle de inundación. La ciudad se encuentra enmarcada por los arroyos Molino, El Curro, de la China y el riacho Itapé, todos afluentes del Río Uruguay (Figura I).

La planta urbana fue delimitada en noviembre de 1958 disponiéndose como límites: al norte el arroyo Curro; al sur el arroyo de la China; al este el arroyo Molino y el riacho Itapé; y al oeste la calle 35 del oeste. Con el paso de los años la ciudad creció y ello ocasiona que se sobrepasen los límites mencionados. Éstos, en su mayoría, están determinados por la red hidrográfica, tanto para la ciudad como para el departamento Uruguay. Actualmente la ciudad se extiende principalmente

2 Proyecto de Investigación y Desarrollo de la Universidad Nacional de Entre Ríos «Caracterización espacial por riesgo ambiental en la ciudad de Concepción del Uruguay con herramientas TIG's». Director: Carñel Griselda E. 
hacia el oeste, ya que tanto hacia el sur como hacia el norte se encuentran las zonas bajas de los arroyos Del Curro y De la China, que limitan el crecimiento urbano.

Entre el río Uruguay y la ciudad se encuentran una serie de islas y zonas bajas anegables que forman parte de su valle de inundación. Más precisamente el frente costero de la ciudad se encuentra sobre el riacho Itapé, que es un brazo del río Uruguay de unos $200 \mathrm{~m}$ de ancho y unos $6 \mathrm{~km}$ de longitud, en el cual confluyen tanto el arroyo del Curro como el arroyo de la China, los que actúan como límites naturales sur y norte al desarrollo urbano.

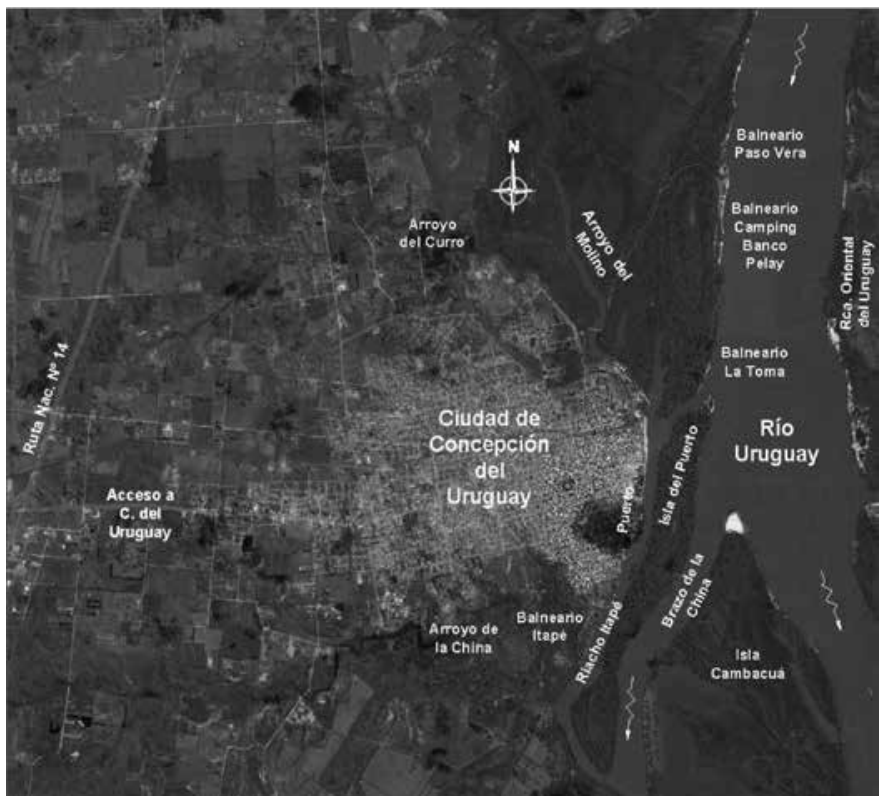

Figura 1. Vista satelital de Concepción del Uruguay y su entorno. Elaboración propia a partir de imagen obtenida del Google Earth consultada en abril de 2012.

El cauce del río Uruguay en esta zona tiene un ancho medio de I, $4 \mathrm{~km}$, variando este entre $800 \mathrm{~m}$ a $2 \mathrm{~km}$, pero su valle de inundación alcanza los $5 \mathrm{~km}$ de ancho, tanto al norte como al sur de la ciudad. A unos $7 \mathrm{~km}$ al norte de la ciudad el valle presenta un estrechamiento con un ancho de solo I,8 km, y desde allí se expande hacia la margen derecha por una zona de bañados, incluyendo el tramo inferior del arroyo del Molino. 
Frente a la ciudad vuelve a angostarse pero hasta un mínimo de unos $3,5 \mathrm{~km}$, para volver a ensancharse al sur, donde comienzan a encontrarse grandes islas dentro del río, como la isla Cambacuá.

El área de estudio, los barrios Cantera 25 y San José, corresponde a la cuenca del arroyo Del Molino (Figura 2). Este arroyo nace en las cercanías de la localidad de Villa San Justo, departamento Uruguay, a los 5 m.s.n.m., con un recorrido de $26 \mathrm{~km}$ hasta su desembocadura en el riacho Itapé, y tiene una cuenca de aporte de 28600 ha. Posee como afluentes principales a los arroyos San Cipriano, Colman, El Curro y El Gato. Desemboca detrás del antiguo hospital, al noreste de la ciudad, en la zona denominada Salamanca, se origina en tres brazos tributarios y posee una cuenca de captación de 250 ha (Brandani, 2000).

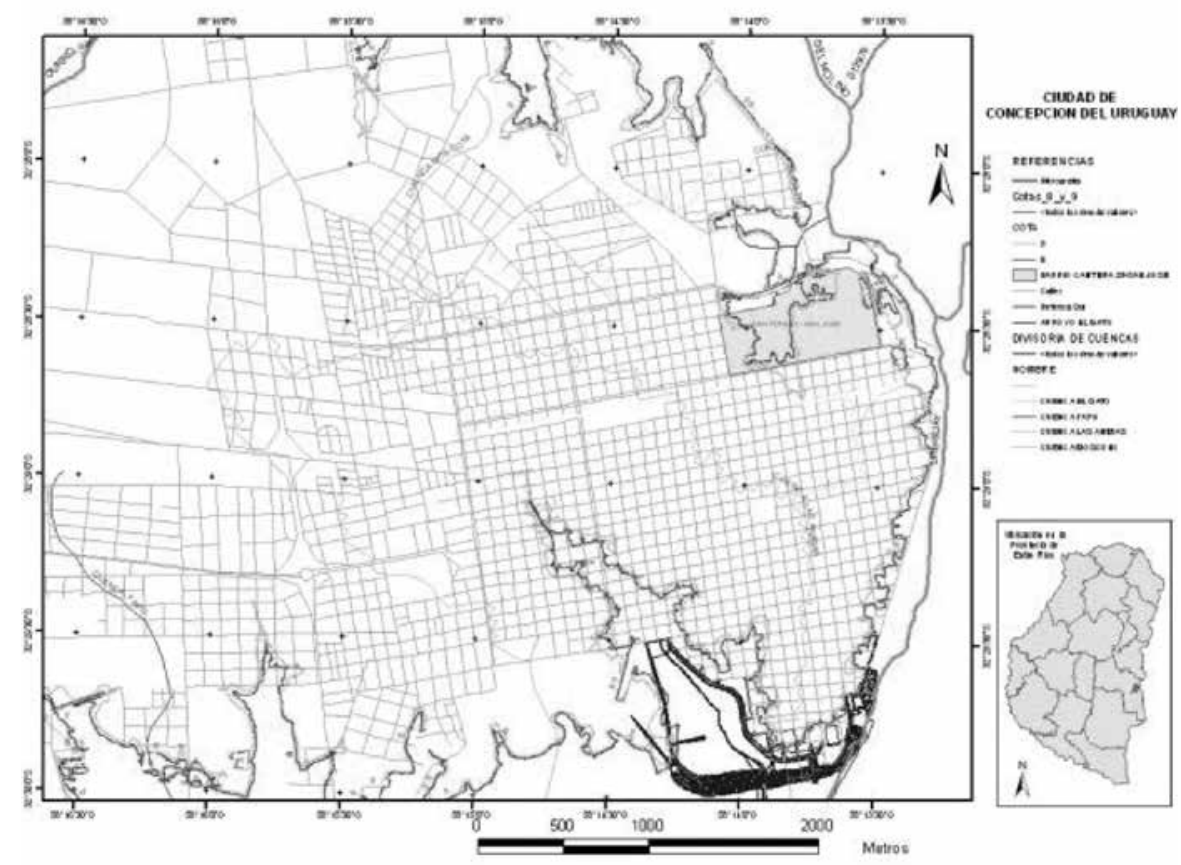

Figura 2. Localización de los barrios Cantera 25 y San José. 
La zona está limitada hacia el sur por una vía estructurante de la ciudad, como es el Boulevard Irigoyen, hacia el oeste por la calle principal Urquiza, al norte por un espacio verde de uso recreativo «La Salamanca» y hacia el este el parque denominado «La Loba».

En Concepción del Uruguay, al igual que en otras ciudades, la limitación al acceso formal de tierras —entre otras causas — ha llevado a la ocupación informal del suelo y a la ubicación de asentamientos mayoritariamente precarios en dichos lugares.

Parte del barrio Cantera 25 se encuentra en una zona baja donde antiguamente funcionaba una cantera, y se corresponde con cotas menores a 8,25 m que es el umbral autorizado para la edificación, por constituir sitios con riesgos de inundaciones.

Sin embargo, actualmente este sector es habitado, por lo que resulta de gran importancia conocer la percepción que la comunidad tiene de una situación de riesgo y las acciones-respuestas que se generan de parte de diferentes actores, como se perciben en relación a las características físicas de las inundaciones y las condiciones socioeconómicas y culturales de su población.

En primera instancia se recopiló información y datos cartográficos y se aplicaron técnicas de Sistema de Información Geográfica (SIG) para determinar zonas inundables.

Para abordar las cuestiones perceptivas se ha adoptado un enfoque integrado que combina metodologías cuantitativas con otras de tipo cualitativo. Como técnicas de recolección de datos se utilizaron encuestas realizadas a la población de los barrios en estudio y entrevistas semiestructuradas a diferentes actores locales y responsables técnicos municipales.

La sistematización de las mismas se efectuó en Microsoft Excel, y fueron procesadas en software ArcGIS io.

Se buscó obtener datos sobre la experiencia del encuestado en relación al riesgo de inundación y valoración de las medidas de gestión existentes y preferencias acerca de las que deberían impulsarse. Así como de las representaciones sociales sobre las amenazas hídricas.

Las encuestas se basaron en un cuestionario de respuestas cerradas y abiertas. Los individuos que formaron parte de la muestra se eligieron a través de un muestreo espacial estratificado sistemático, ya que se realizó con base en una zonificación de áreas previas, y atendiendo a ciertos criterios:

- La localización de aproximadamente la mitad de los encuestados corresponde a sectores inundables. 
- La otra mitad se considera en área inmediata, afectados también por la inundación pero que no han vivido situaciones de relocalización, considerando que igualmente son afectados por las consecuencias derivadas de la inundación en su salud y en la afectación que se produce en el ambiente.

Esto conlleva a una distribución territorial de la población encuestada de tres a cuatro ( 3 a 4 ) familias por manzana para asegurar la representatividad de la población territorialmente. Se trabajó mediante una muestra estadística, cuyo tamaño (I5o encuestas) fue obtenido por una calculadora de muestras online. ${ }^{3}$ Para conseguir el tamaño muestral, se aceptó un margen de error del $6 \%$, nivel de confianza del $90 \%$, nivel de heterogeneidad del $30 \%$, siendo 2830 el tamaño del universo a encuestar.

La guía para las entrevistas semi estructuradas fueron diseñadas considerando datos generales sobre la inundación en la ciudad y en especial en el área de estudio.

\section{Desarrollo y resultados}

Mediante la utilización del SIG se hizo una zonificación del sector afectado por la inundación en la ciudad de Concepción del Uruguay. El área de estudio, barrios Cantera 25 y San José, presentó una población en riesgo por inundación de 2830 habitantes aproximadamente (Censo 2010).

Ciento cincuenta ( 150 ) encuestas se realizaron durante los meses de marzo y abril de 2013 y las entrevistas semi estructuradas fueron realizadas a seis (6) funcionarios de la Municipalidad, a tres (3) Concejales, a un representante de Defensa Civil y a un representante de la Cruz Roja.

Percepción de los habitantes en riesgo de inundación (encuestas)

El $65 \%$ de los encuestados posee más de 40 años de edad, y poco más de la mitad tiene una antigüedad en los barrios de más de 20 ańos, lo cual significa un sentimiento de pertenencia a los mismos. El 49 \% respondió

3 http://www.netquest.com/panel_netquest/calculadora_muestras.php 
que se inundó alguna vez su vivienda, fenómeno buscado al realizar las encuestas con una distribución territorial definida por las cotas de inundación y selección de habitantes por manzana, a los efectos de garantizar la comparación entre quienes han sido afectados directa o indirectamente con el fenómeno.

Tabla 1. Categorización sobre las amenazas a que está expuesta el área de estudio.

\begin{tabular}{|c|c|c|}
\hline Tipología & $\begin{array}{l}\text { Cantidad } \\
\text { (más de una } \\
\text { respuesta por } \\
\text { encuestado) }\end{array}$ & $\begin{array}{l}\text { Cantidad } \\
\text { (prioridad, sólo } \\
\text { una respuesta por } \\
\text { encuestado) }\end{array}$ \\
\hline \multicolumn{3}{|l|}{ AMENAZAS NATURALES } \\
\hline $\begin{array}{l}\text { Inundaciones por creciente o desborde } \\
\text { del río }\end{array}$ & 104 & 93 \\
\hline Tormentas / granizada & 104 & 32 \\
\hline Vientos fuertes & 50 & 1 \\
\hline Sequías & 6 & 0 \\
\hline \multicolumn{3}{|l|}{ AMENAZAS SOCIONATURALES } \\
\hline Deslizamientos de tierra & 4 & 0 \\
\hline Erosión de la franja ribereña & 2 & 0 \\
\hline \multicolumn{3}{|l|}{ AMENAZAS ANTRÓPICAS } \\
\hline Incendios & 2 & 0 \\
\hline Accidentes industriales / explosiones & 0 & 0 \\
\hline Accidentes de tránsito & 4 & 0 \\
\hline $\begin{array}{l}\text { Derrames de sustancias tóxicas, químicas } \\
\text { o peligrosas }\end{array}$ & 3 & 1 \\
\hline Contaminación ambiental & 47 & 9 \\
\hline Otra & 3 & 0 \\
\hline Sin datos & 14 & 14 \\
\hline
\end{tabular}

En cuanto a las cuestiones relacionadas con la percepción frente al riesgo de inundaciones, se obtuvieron los resultados que se comentan a continuación. En la Tabla I se detalla las amenazas que los encuestados consideran más importantes como causantes de pérdidas económicas en su barrio, siendo éstos las inundaciones producto de las crecientes del río y los fenómenos meteorológicos.

Del total de individuos que se han inundado alguna vez, el $89 \%$ considera a las inundaciones como el fenómeno principal que afecta la vivienda o el barrio, mientras que los que no han resultado afectados directamente, es decir aquellos que no se han inundado nunca, consideran prioritario la ocurrencia de tormentas/granizadas, en segundo 
lugar las inundaciones y en tercer lugar aspectos que tienen que ver con la contaminación ambiental, deficiencia de la infraestructura y el mejoramiento del espacio público.

$\mathrm{Al}$ preguntar acerca de cada cuántos ańos consideran que se producen las inundaciones, el $75 \%$ de la población estima que las inundaciones periódicas se producen entre i a 5 años, lo cual es ajustado a la realidad, considerando la cota de inundación entre 5 y $6 \mathrm{~m}$, según datos aportados por Prefectura Naval Argentina.

El $50 \%$ de la población encuestada considera que la ausencia de infraestructura de protección es la causante principal de las inundaciones en las viviendas, y solamente el 21\% acusa a «la ubicación de las viviendas en zonas inundables».

De la población inundada, el $64 \%$ ha sufrido entre i y 4 veces inundación de su vivienda, y el $36 \%$ por encima de dicho valor, lo que supone cierta experiencia frente al mismo.

Respecto de los lugares de evacuación durante los períodos de inundación (Tabla 2) se observa que el $55 \%$ de la población que se ha inundado, recibe contención de familiares, y el $23 \%$ se traslada a diferentes refugios como la Capilla San José, ubicada en el barrio San José, la escuela $\mathrm{N}^{\circ} 58$ Santiago del Estero, instalaciones del Ferrocarril, Escuela $\mathrm{N}^{\circ}$ 93, Jardín de infantes «Tortuguitas», Club Lanús, CEF $\mathrm{N}^{\circ}$ I2 y Centro de Integración Comunitaria (CIC).

Tabla 2. Sitios de evacuación durante eventos de inundación.

\begin{tabular}{lrrr}
\hline \multirow{2}{*}{ Opciones } & \multicolumn{2}{l}{$\begin{array}{l}\text { Cantidad } \\
\text { (más de una respuesta } \\
\text { por encuestado) }\end{array}$} & \multicolumn{2}{l}{$\begin{array}{l}\text { Cantidad } \\
\text { (prioridad, sólo una } \\
\text { respuesta por encuestado) }\end{array}$} \\
\hline A casa de familiares & 41 & 41 \\
\hline A casa de vecinos & 2 & 1 \\
\hline Refugios (cuáles) & 18 & 17 \\
\hline Otro & 17 & 14 \\
\hline Sin datos & 1 & 1 \\
\hline
\end{tabular}

Otra de las respuestas obtenida fue «no nos trasladamos», lo que permite inferir que se han resguardado en sus viviendas por temor a perder las pertenencias por posibles robos o por efectos del mismo fenómeno. 
Al indagar sobre las causas de su regreso al área afectada, el $39 \%$ lo hace por temor a perder sus pertenencias, seguido de la falta de recursos para mudarse a un lugar más seguro (un $24 \%$ ). La vivienda, más allá de sus condiciones de precariedad es determinante para regresar al área y esto se relaciona con la falta de recursos y de posibilidades de adquirir suelo y vivienda correctamente localizada en un sector ambientalmente seguro.

Con respecto al alerta a la población en general, casi la mitad de los encuestados ha señalado que es la propia experiencia la que permite alertar frente a una inundación, gran parte no ha recibido alerta o información, y algunos pocos son alertados por vecinos y familiares. Los resultados demuestran una escasa injerencia de la Municipalidad y otros organismos para alertar a la población.

Con relación a las consecuencias de las inundaciones, la variedad de estas respuestas habla de un efecto multicausal de las inundaciones, que irrumpe en varias áreas de la vida de las personas en forma de daños materiales y aparición de vectores que transmiten en enfermedades como los mosquitos y parásitos, seguidas de afectaciones psicológicas y de salud.

Sin embargo, otras respuestas obtenidas, demuestra que el evento ocasiona numerosos problemas ambientales, económicos, sociales y de salud de la población, variando las respuestas de acuerdo a la afectación que ha percibido cada uno de los encuestados en su calidad de vida.

Los padecimientos más comunes en ambas cuencas durante y después de las inundaciones fueron infecciones cutáneas, enfermedades respiratorias, infecciones gastrointestinales, dengue y paludismo. Es de especial atención el caso de estas dos últimas, ya que la propagación de los mosquitos portadores se debe al estancamiento de agua y al tiempo que tarda en drenar. Las enfermedades a las que se exponen las personas son variadas y representan graves riesgos potenciales. Por lo tanto, el papel que desempeñan los servicios de salud en la vulnerabilidad de una población es significativo.

Como medida primordial para evitar o prevenir el fenómeno de inundaciones, el 9i \% de la población considera prioritario la construcción una defensa de contención del río, y un mínimo porcentaje, solicita un plan de emergencia, educación comunitaria, reubicación de las viviendas en zonas no inundables y manejo de la cuenca.

Poco más del $90 \%$ de los habitantes encuestados no tiene pensado o programado trasladarse del lugar. Ello se debe a la falta de recursos econó- 
micos para mudarse a un sitio más seguro, por sus propiedades actuales (vivienda, negocios), por la cercanía a la zona céntrica de la ciudad, porque no se han inundado, es decir, viven en la zona más alta del barrio, y porque creen que se realizarán obras de protección (defensa norte).

Solamente el $20 \%$ de las personas encuestadas participan en acciones comunitarias que promuevan soluciones para el problema de la inundación. Estas integran o forman parte de las reuniones barriales que se realizan en la capilla o en SUM (Salón de Usos Múltiples) del barrio Cantera 25, juntas barriales y reuniones de la comisión propulsora de la defensa norte.

Esto podría estar sugiriendo que el riesgo hídrico no es una prioridad en la vida diaria de cada habitante del barrio y también que la preocupación frente al problema descansa en una posible solución de tipo ingenieril y/o estatal.

Al preguntar si algún funcionario del gobierno se ha contactado con los habitantes para tratar algún tema relacionado con las inundaciones, solo el II \% ha respondido positivamente. Éstos señalaron que han obtenido asistencia luego de una inundación por parte del municipio mediante la entrega de abrigos, asistencia económica de la CAFESG (Comisión Administradora para el Fondo Especial de Salto Grande), campaña de limpieza luego de una inundación, vacunas y ayuda en recursos humanos. Para algunos, los funcionarios solo se acercan en épocas de elecciones para hacer promesas, y no dan soluciones definitivas.

Sobre la localización en un primer momento de la población en dichas áreas, la mayoría de las respuestas por parte de aquellos que se han inundado, fueron por cuestiones económicas. Fundamentan en la falta de recursos económicos para adquirir terrenos en otros lugares, porque eran terrenos gratuitos, baldíos del municipio, por herencia de sus familiares y su cercanía a la zona céntrica.

Algunos de los que no se han inundado, y que habitan la zona más alta de los barrios, señalan que las personas inundadas se han localizado en la zona por conveniencia, ya que al ser terrenos fiscales no debían pagar, y por la asistencia que se les otorga. 
Percepción de funcionarios y dirigentes sociales (entrevistas)

Del procesamiento del total de once (II) entrevistas a funcionarios, concejales y representantes de ONG, se determinan los siguientes resultados:

Poco más del $90 \%$ afirma que las inundaciones por crecida del río es el principal evento que afecta al barrio Cantera 25. La mayoría declara que la principal causa es la incorrecta ubicación de las viviendas y el restante la ausencia de una defensa de protección. Poco más de la mitad, afirma que la población regresa al área por la existencia de su vivienda, seguida por la falta de recursos para mudarse a un lugar más seguro y por conveniencia económica debido a que se ubican en áreas cercanas al centro de la ciudad. La mayoría señaló que las organizaciones comunitarias y sitios de mayor importancia lo constituyen el Salón de Usos Múltiples (SUM) y el comedor comunitario «Mis soles». La representante de Cruz Roja indicó: "hay líderes barriales que no trabajan asociados y responden a diferentes intereses. Las familias no están integradas, y eso puede deberse a que no pertenecen al mismo lugar. No tienen capacidad de ayudarse entre sí, no hay identidad».

En cuanto a la pregunta acerca de si tienen conocimiento de la existencia de reuniones informativas con los habitantes en las zonas en riesgo para ilustrar que se debe hacer en caso de emergencia, el 9r \% respondió de manera positiva. Algunos, como la directora de Ambiente Sustentable y el subsecretario de Planeamiento Urbano municipales, respondieron que los encargados son los bomberos, la policía, Defensa Civil y la Secretaría de Salud, pero que desconocen de qué se tratan esas reuniones.

El subsecretario de Obras Públicas indicó que desde la Secretaría de Desarrollo Social se atiende a los vecinos conforme a la emergencia del reclamo y no sólo debido a la problemática de las inundaciones.

Asimismo, el secretario de Desarrollo Social afirmó: «Desde el municipio junto con Defensa Civil y Cruz Roja se trabaja en conjunto. Se cuenta con un programa de prevención de siniestros, en el cual se ha informado a los habitantes en riesgo y se han hecho relevamientos de las familias que viven allí para conocer entre otras cosas las familias más vulnerables». La representante de Cruz Roja ha señalado que desde esta entidad se hacen reuniones con los vecinos del barrio informándoles que deben hacer en caso de emergencia, pero señala también que «ellos ya saben qué hacer cuando crece el río». 
El coordinador general de Defensa Civil de la provincia de Entre Ríos expresó que el Instituto Nacional de Agua (INA) y la Comisión Técnica Mixta de Salto Grande (CTM) brindan información con respecto al comportamiento del río y previo a la crecida y que el director local de Defensa Civil hace reuniones con los habitantes.

El encargado de protección marítima y puertos, y ayudante de Tercera en Prefectura de Concepción del Uruguay señaló que se cuenta con un plan de contingencia tanto desde Prefectura como de la Municipalidad.

Dos de los tres concejales entrevistados dijeron que se realizan reuniones barriales y de participación ciudadana, y el otro ha señalado que "personal de Defensa Civil, Salud y Cruz Roja indican donde están los refugios y las medidas de protección que deben tomar cuando hay alerta por inundaciones».

El secretario de Servicios sanitarios y desagües pluviales fue el único que dijo desconocer de la existencia de reuniones informativas con los habitantes del barrio para informar qué hacer en caso de emergencia.

Con respecto a la pregunta acerca de adónde creen que se trasladan los afectados cuando se producen inundaciones, en primer lugar, la mayoría de los entrevistados respondió que se traslada a refugios/clubes, siguiéndole la casa de familiares, dependencias municipales, provinciales, nacionales, SUM (salón de usos múltiples), y por último a casa de vecinos. Dentro de los refugios nombrados se encuentran la Escuela Santiago del Estero, SIC, centro de deportes, Club Lanús, y otros ubicados en el barrio La Concepción. La representante de Cruz roja agregó que «los habitantes de menos recursos van a los centros de evacuados, los de mejores condiciones alquilan".

A la pregunta sobre si tienen conocimiento de las medidas adoptadas por los habitantes del barrio ante el alerta de inundación, el $36 \%$ respondió de manera afirmativa. Entre las respuestas cabe destacar que los afectados saben a qué altura del río tienen que evacuar, y una de las obtenidas fue "para saber si se van a inundar ponen una rama y ven cuánto va creciendo».

Las familias que cuentan con algún recurso económico, contratan fletes para el traslado de las pertenencias, mientras que las de bajos recursos esperan los camiones del municipio o ayuda de defensa civil. Otras familias se trasladan a casa de familiares o centros de evacuados, y en la mayoría de los casos uno de los miembros se resguarda en la casa para su cuidado y evitar el robo de las pertenencias. 
En cuanto a qué se debería hacer en el barrio con la ocurrencia de las inundaciones, poco menos del $50 \%$ respondió que debería realizarse une defensa norte, seguido por la educación comunitaria, reubicación de las viviendas, realizarse un plan de emergencia, y manejo de la cuenca. "Que se respeten las normas urbanísticas» fue la respuesta del subsecretario de Planeamiento Urbano.

Respecto de quién creen que influye en mayor medida en el alerta de las inundaciones en la ciudad, las respuestas fueron variadas, no predominó una sobre otra: la propia experiencia junto a la autoridad municipal fueron las principales.

Todos los entrevistados respondieron de manera positiva acerca de la existencia de registros y la identificación de las zonas inundables de la ciudad y añadieron que hay un censo realizado con las zonas en riesgo. El secretario de Desarrollo Social agregó que «desde acción social se identifican no sólo los barrios sino los integrantes de las familias y se ve qué dificultades tienen en caso de siniestros para poder asistirlos».

En cuanto a las consecuencias que creen que acarrean las inundaciones, el 9r \% de las respuestas fueron daños materiales en las viviendas, seguido por daños psicológicos, aparición de vectores transmisores de enfermedades, deterioro de las calles y de la infraestructura. Otra de las respuestas fue afección a la salud debido al agua que se mezcla con las excretas de los pozos negros.

Todos los entrevistados, a excepción de uno, indicaron que el gobierno municipal realiza evaluaciones de daños y pérdidas por inundaciones en la ciudad, y se les ha provisto de dinero a los afectados en las últimas inundaciones.

Cuando se preguntó si creen que existe una adecuada coordinación entre las autoridades locales y organismos de la sociedad civil para responder en caso de emergencia, todos los entrevistados respondieron de manera positiva. Asimismo, el secretario de Desarrollo Social agregó: «si bien existe una adecuada coordinación, hay que afinar, especificar más el rol de cada entidad, ver en detalle que le corresponde a cada uno. Eso influye en el trato de los inundados ya que, por ejemplo, al juntar las pertenencias en los camiones de diferentes familias puede traer disgustos».

A la pregunta sobre si conocen de la existencia de un presupuesto asignado específicamente para amenazas naturales, las respuestas fueron ambiguas y confusas. 
Los más cercanos a la cuestión de las inundaciones, para estos entrevistados fueron la Secretaría de Desarrollo Social, seguida de la Secretaría de Salud y Obras Públicas, Secretaría de Ambiente Sustentable, Secretaría de Servicios sanitarios y desagües pluviales, y por último Defensa Civil. Con referencia a la solución posible para que no haya afectados por las inundaciones, las respuestas fueron diversas: construcción de una defensa norte, no urbanizar zonas inundables, una correcta planificación urbana, concientización de la gente, traslado de las personas, programas de prevención, plan de emergencia y colaboración de los vecinos para no obstruir las infraestructuras.

\section{Discusión}

Es importante señalar que de los encuestados que han sentido los efectos de una inundación, la mayoría es consciente de la posibilidad de que ocurra nuevamente el fenómeno, como también de sus posibles consecuencias. Ello remite a un escenario complejo y que tiene que ver con cuestiones económicas y sociales que imposibilitan el acceso formal al suelo y vivienda, resultado de políticas de exclusión social. Muchos de ellos señalan que la responsabilidad es del gobierno debido a la falta de eficacia en las decisiones y acciones adoptadas, y demandan como primera medida para evitar nuevos daños la construcción de obras de contención. De los resultados obtenidos se desprende que existe una gran confianza en las obras estructurales como solución al problema de las inundaciones, y es probable que el hecho de que la zona sur de la ciudad no haya sufrido daños durante la última inundación gracias a una obra estructural sea clave en las representaciones de los encuestados. Eventualmente, en el momento previo, durante o después del desenlace del fenómeno, la población potencialmente afectada es más consciente de la naturaleza del riesgo hídrico. Los que han resultado perjudicados directamente, señalan que la alerta o información acerca del desarrollo del fenómeno provenía de su propia experiencia, dado el conocimiento que se tiene sobre el comportamiento del río o los efectos de las lluvias sobre el cauce del arroyo, familiares o vecinos, con un porcentaje mínimo de los bomberos/Defensa civil y del Estado municipal, lo que manifiesta insuficiente vinculación del gobierno con la comunidad, notando diferencias entre lo seńalado por los funcionarios y la comunidad. Esta investigación sobre percepción de riesgos muestra que los 
funcionarios y/o concejales definen el riesgo de una manera técnica y restringida a las posibilidades de la función que representan, excepto desde la Cruz Roja, se observa una actitud más comprometida con la realidad social y cultural y conocimiento de las conductas de los habitantes del área frente al problema, mientras que los encuestados consideran el riesgo influidos por factores psicológicos, sociales, institucionales y culturales.

Analizando la normativa existente, el Código Urbano de Concepción del Uruguay define a este sector de la ciudad como área residencial con infraestructura incompleta. Ante esta potencialidad, el 9r \% de la población encuestada demanda la concreción del proyecto para contener las inundaciones, denominado «Defensa norte», como «soluciones definitivas». Se ha comprobado con una defensa existente en la ciudad, "Defensa sur", que no es suficiente para mejorar la calidad de vida. Pese a esto, los reclamos en búsqueda de concreción del proyecto son cada día más notorios. En este sentido, el papel del Estado no debe centrarse en la instalación de obras de ingeniería, sino por el contrario establecer políticas adecuadas de inclusión social y una planificación del territorio que contemple aspectos urbanísticos, ambientales, económicos y sociales. 


\section{Referencias bibliográficas}

Arrillaga, H.; Grand, M. L. y Busso, G. (2009).

Vulnerabilidad, riesgo y desastres. Sus relaciones de causalidad con la exclusión social en el territorio urbano santafesino. En Herzer, H. y Arriaga H. (comps.), La construcción social del riesgo y el desastre en el aglomerado Santa Fe. Santa Fe: Ediciones UNL.

Brandani, A. (200I). Programa de Asistencia Técnica para el Manejo Ambiental del Área Urbana en la ciudad de Concepción del Uruguay. SUPCE Provincia de Entre Ríos. Buenos Aires.

Burton, I. y Kates R. (1972). The perception of natural hazard in resource management. En Ward, P. y Mayfield, R. (eds.), Man, space and environment. Oxford: Oxford University Press.

Calvo García-Tornel, F. y Granell, M. C. (2009).

Valoración social del riesgo por inundación en el litoral meridional de la región de Murcia. Scripta Nova, XIII(295).

Masi, M. B. y Kessler, M. E. (2009). El entramado asociativo en la ciudad de Santa Fe: vulnerabilidad y eficiencia de los lazos sociales en un contexto de crisis. En Herzer, H. y Arriaga H. (comps.) La construcción social del riesgo y el desastre en el aglomerado Santa Fe. Santa Fe: Ediciones UNL.

Rovira A, (1986). Percepción del riesgo de inundaciones en la Comuna de San Bernardo. Depto. de Geografía, Universidad de Chile.

Smith, K. y Tobin, G. (1979). Human adjustment to the flood hazard, topics in applied geography. London: Logman Group Limited.

Urquiza Almandoz, O. F. (2002). Historia de Concepción del Uruguay. Entre Ríos: Comisión Técnica Mixta de Luis e Salto Grande.

Ward, R. (1978). Focal problems in geography. Floods, a geographical perspective. Gran Bretańa. 